\title{
p-Doped Graphene/Graphitic Carbon Nitride Hybrid \\ Electrocatalysts: Unraveling Charge Transfer Mechanisms for Enhanced Hydrogen Evolution Reaction Performance
}

\author{
Xin Tan, Hassan A. Tahini, Sean C. Smith* \\ Integrated Materials Design Centre (IMDC), School of Chemical Engineering, UNSW Australia, \\ Sydney, NSW 2052, Australia
}

*Corresponding author: sean.smith@,unsw.edu.au 


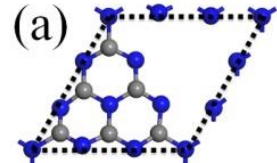

$a=6.93 \AA$

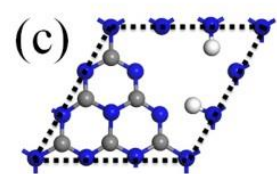

$a=7.10 \AA$

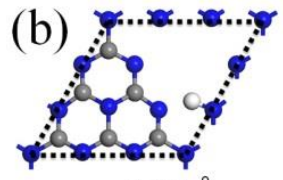

$a=7.00 \AA$

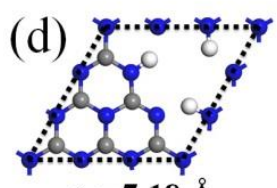

$a=7.18 \AA$

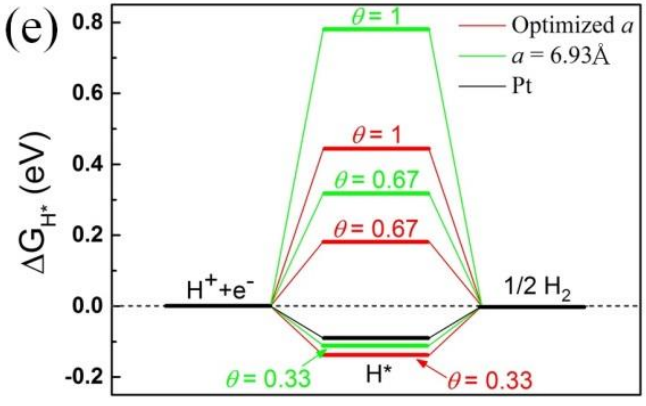

Reaction coordinate

Figure S1. Top (upper) and side (lower) views of the lowest-energy configurations of pure $g-\mathrm{C}_{3} \mathrm{~N}_{4}$ monolayer with $\mathrm{H}^{*}$ coverage (a) $\theta=0$, (b) $\theta=0.33$, (c) $\theta=0.67$, and (d) $\theta=1$. The blue, grey and white balls represent $\mathrm{N}, \mathrm{C}$ and $\mathrm{H}$ atoms, respectively. The unit cells are indicated by black dot lines, and the optimized lattice constants are listed below. (e) The calculated free energy diagram of HER at the equilibrium potential for pure $g-\mathrm{C}_{3} \mathrm{~N}_{4}$ monolayer under different $\mathrm{H}^{*}$ coverage $(\theta=0,0.33,0.67$ and 1$)$. The free energy diagram of HER for Pt is also shown for comparison. 

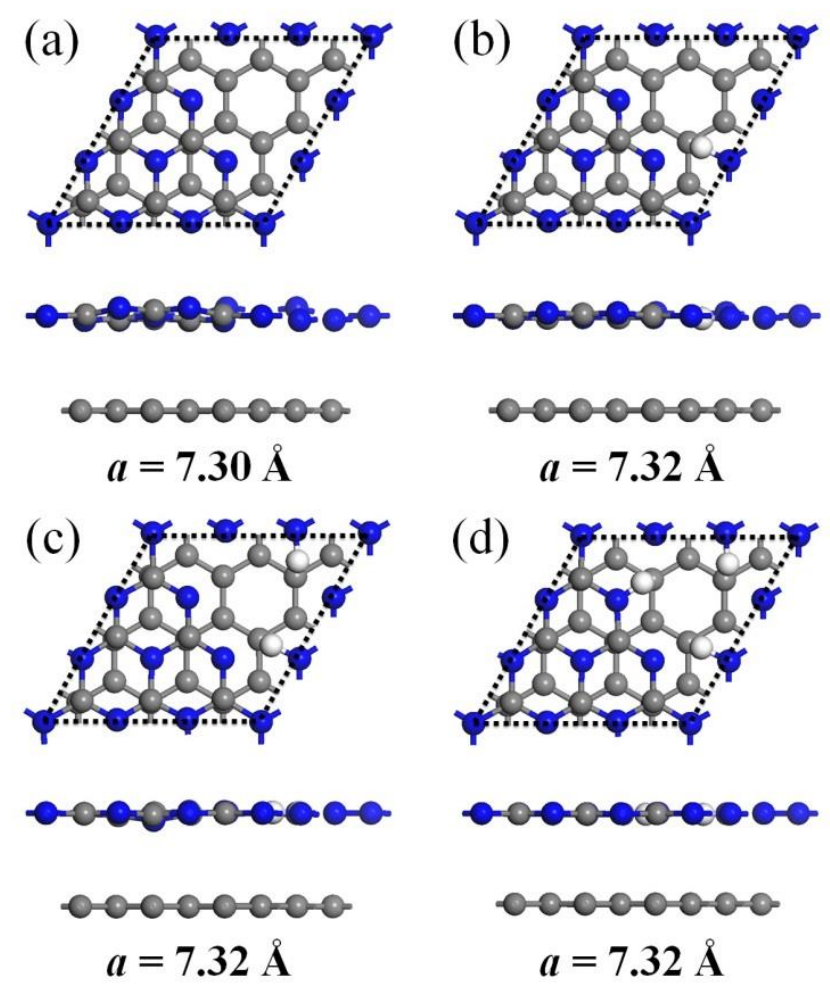

Figure S2. Top (upper) and side (lower) views of the lowest-energy configurations of $g-\mathrm{C}_{3} \mathrm{~N}_{4} @$ G hybrid with $\mathrm{H}^{*}$ coverage (a) $\theta=0$, (b) $\theta=0.33$, (c) $\theta=0.67$, and (d) $\theta=1$. The blue, grey and white balls represent $\mathrm{N}, \mathrm{C}$ and $\mathrm{H}$ atoms, respectively. The unit cells are indicated by black dot lines, and the optimized lattice constants are listed below. 

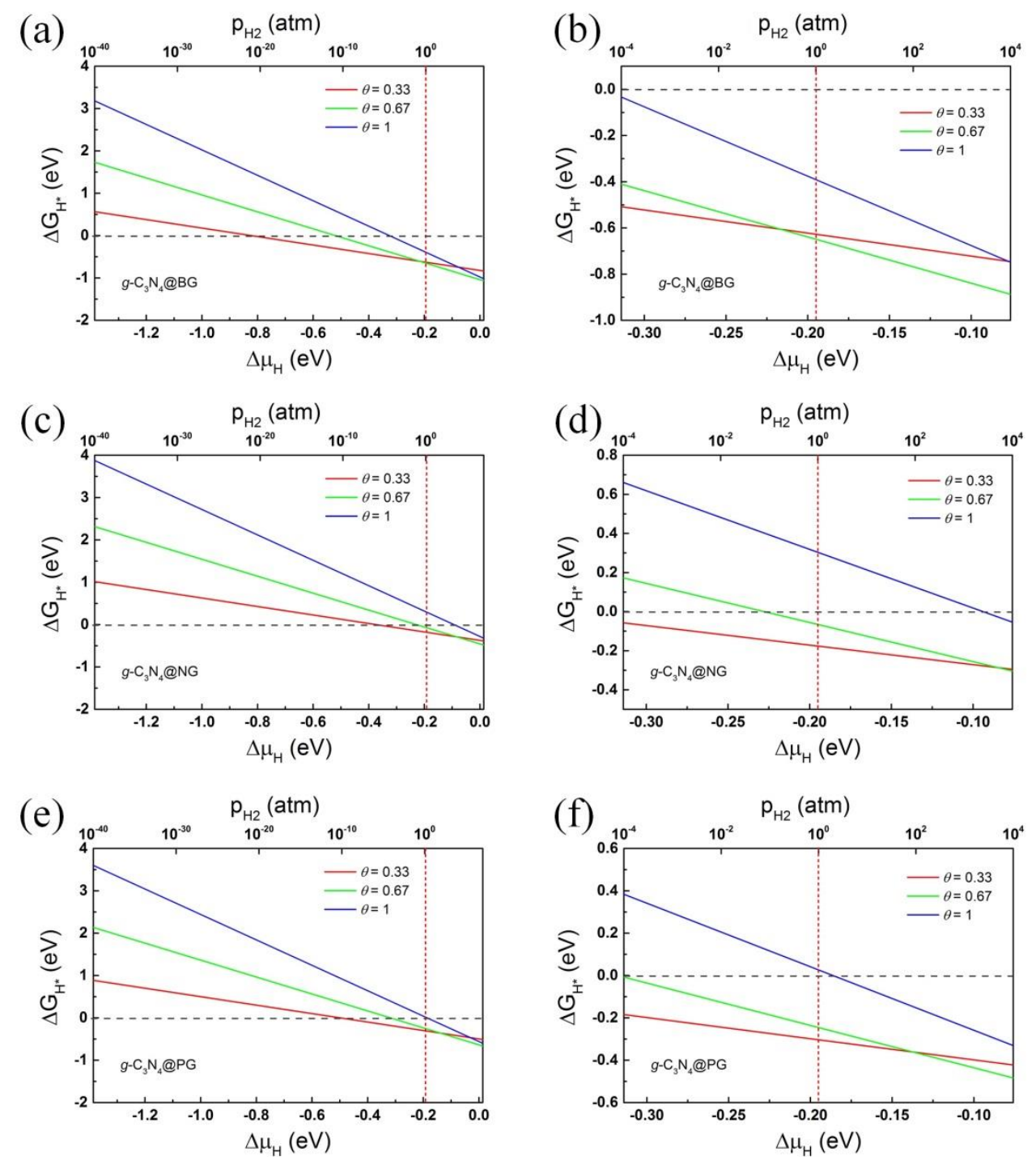

Figure S3. Gibbs free energy of adsorption at $298 \mathrm{~K}$ as a function of hydrogen chemical potential $\left(\Delta \mu_{H}\right)$ or $\mathrm{H}_{2}$ pressure for (a), (b) $g-\mathrm{C}_{3} \mathrm{~N}_{4} @ \mathrm{BG}, \quad$ (c), (d) $g-\mathrm{C}_{3} \mathrm{~N}_{4} @ \mathrm{NG}$, and (e), (f) $g-\mathrm{C}_{3} \mathrm{~N}_{4} @ \mathrm{PG}$ hybrids with different $\mathrm{H}^{*}$ coverages: (a), (c), (e) $\Delta \mu_{H}$ from -0.139 to $0.013 \mathrm{eV}$; (b), (d), (f) $\Delta \mu_{H}$ from -0.314 to $-0.076 \mathrm{eV}$. 

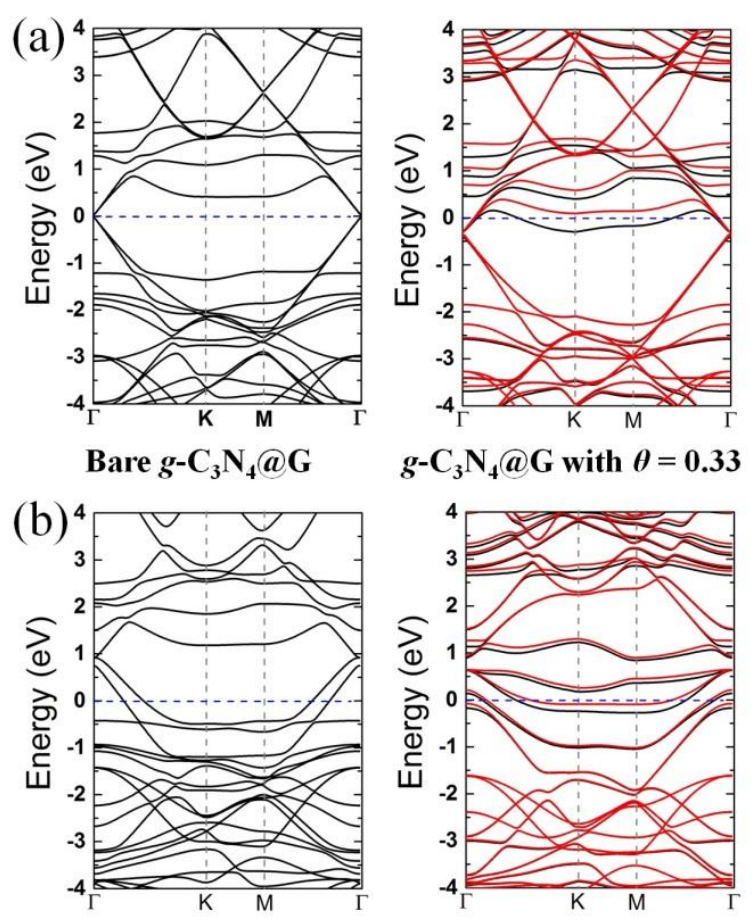

Bare $g-\mathrm{C}_{3} \mathrm{~N}_{4} @ B G$

$g-\mathrm{C}_{3} \mathrm{~N}_{4} @ \mathrm{BG}$ with $\theta=0.67$
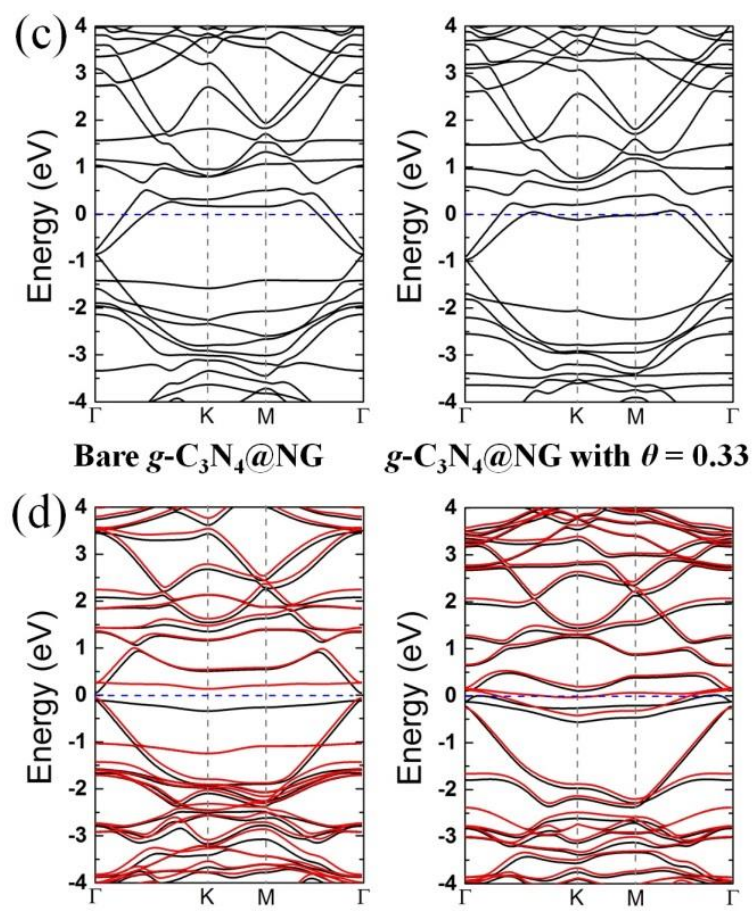

Bare $g-\mathrm{C}_{3} \mathrm{~N}_{4} @ P G$

Figure S4. The calculated band structures of (a) $g-\mathrm{C}_{3} \mathrm{~N}_{4} @ \mathrm{G}$, (b) $g-\mathrm{C}_{3} \mathrm{~N}_{4} @ \mathrm{BG}$, (c) $g-\mathrm{C}_{3} \mathrm{~N}_{4} @ \mathrm{NG}$, and (d) g- $\mathrm{C}_{3} \mathrm{~N}_{4} @ \mathrm{PG}$ hybrids with/without $\mathrm{H}^{*}$ adsorption. The blue dashed line denotes the Fermi level. The red and black lines in (a), (b) and (d) denote the spin-up and spin-down states, respectively. 